\title{
Comportamiento anticorrosivo de pinturas de base agua aplicadas en aceros
}

\author{
M. BETHENCOURT, F.J. BOTANA, M.J. CANO, R.M. OSUNA, M. MARCOS ${ }^{1}$
}

Dpto. de Ciencia de los Materiales e Ingeniería Metalúrgica y Química Inorgánica. Univ. de Cádiz. Facultad de Ciencias del Mar. Puerto Real, 11510, Cádiz. 'Departamento de Ingeniería Mecánica y Diseño Industrial. Universidad de Cádiz. Escuela Superior de Ingeniería. 11003 Cádiz.

\begin{abstract}
Los recubrimientos orgánicos, especialmente las pinturas, son uno de los métodos de protección más ampliamente empleados para evitar la corrosión del acero. Su bajo coste y facilidad de aplicación son unas de las principales razones por las que, aproximadamente, el $90 \%$ de la totalidad de las superficies metálicas se protegen con pinturas. En este trabajo se estudia el comportamiento de una pintura acrílica de base agua aplicada sobre un acero de uso frecuente en la Industria Naval. Para dicho estudio, las muestras pintadas se sometieron a ensayos electroquímicos de Polarización Lineal y Espectroscopía de Impedancia Electroquímica en disolución de $\mathrm{NaCl}$ al 3.5\%, tras diferentes periodos de inmersión en este medio.
\end{abstract}

Palabras Clave: Pintura base agua, Acero, Resina acrilica, Polarización Lineal, EIS

\section{Waterborne Paints Anticorrosive Behaviour on Steels}

Organic coatings, mainly paints, are one of the most important methods for protecting steel against corrosion. Both their low cost and easy applicability are between the reasons for selecting them as a method for protecting approximately $90 \%$ of the structures exposed to atmosphere. In this work, anticorrosive properties of an acrylic waterborne paint applied to a naval steel have been evaluated in $\mathrm{NaCl}$ at $3.5 \%$ solutions by means of electrochemicals techniques, such as Linear Polarisation and Electrochemical Impedance Spectroscopy.

Keywords: Waterborne paint, Steel, Acrylic resin, Linear Polarisation, EIS

\section{INTRODUCCIÓN}

Aproximadamente el $90 \%$ de la totalidad de las superficies metálicas se protegen con pinturas [1]. Tradicionalmente se han utilizado pinturas formuladas con disolventes orgánicos. Sin embargo, la necesidad de reducir la emisión de compuestos orgánicos volátiles (VOC), perjudiciales para la atmósfera, ha permitido el desarrollo de pinturas ecológicas que emplean como disolvente el agua.

En este trabajo se estudia el comportamiento de una pintura acrílica de base agua, no agresiva con el medio ambiente, aplicada sobre un acero de uso preferente en la industria naval. Para ello se han empleado técnicas electroquímicas, como el seguimiento del potencial a circuito abierto (OCP) y la polarización lineal (LP) para evaluar el deterioro del recubrimiento. Por otro lado, mediante EIS y haciendo uso del circuito simple que representa la respuesta de un metal pintado, Figura 1, se han obtenido valores de la entrada de agua en la pintura [2].

\section{EXPERIMENTAL}

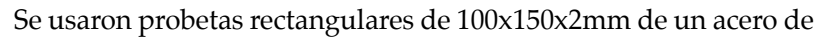
uso naval DIN St 35.8 y se sometieron a un preparado superficial tipo chorreado Sa 3. Seguidamente, tras desengrasar con acetona, se procedió a aplicar la pintura acrílica sobre las probetas chorreadas haciendo

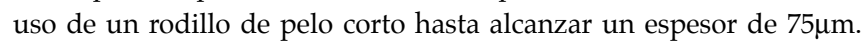
Posteriormente, se evaluó la capacidad protectora del recubrimiento. Para ello, las probetas fueron sometidas a ensayos de inmersión durante diferentes periodos en disolución de $\mathrm{NaCl}$ al $3.5 \%$, tras los cuales se le realizaron ensayos electroquímicos de OCP, LP y espectroscopía de impedancia electroquímica (EIS). Se usó una celda cilíndrica fijada

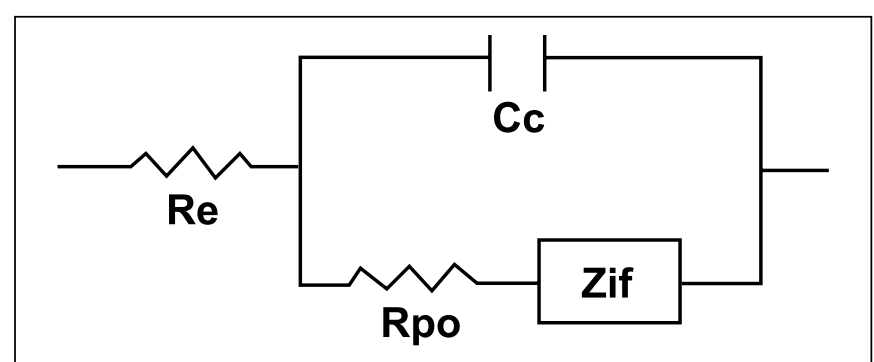

Figura 1. Circuito equivalente para un metal pintado

al electrodo de trabajo con un área expuesta de $13 \mathrm{~cm}^{2}$ a la disolución $3.5 \%$ de $\mathrm{NaCl}$ con un SCE como electrodo de referencia. Las medidas se efectuaron haciendo uso de un potenciostato SI 1287 de Solartron al que se acopló un analizador de respuesta de frecuencia (FRA) de Solartron modelo SI 1255 para los ensayos de EIS.

\section{RESULTADOS Y DISCUSIÓN}

\subsection{Evolución del potencial de corrosión.}

En la Figura 2 se muestra la evolución del Potencial de Corrosión en la disolución de $\mathrm{NaCl}$ de una muestra pintada. Como puede observarse, existe una tendencia a valores más activos durante los tres primeros días, lo que indica que la proporción de área superficial catódica respecto a la anódica disminuye y resulta significativo el proceso de corrosión bajo el recubrimiento. En los días siguientes el potencial de corrosión se desplaza hacia valores más nobles. Esto implica que 


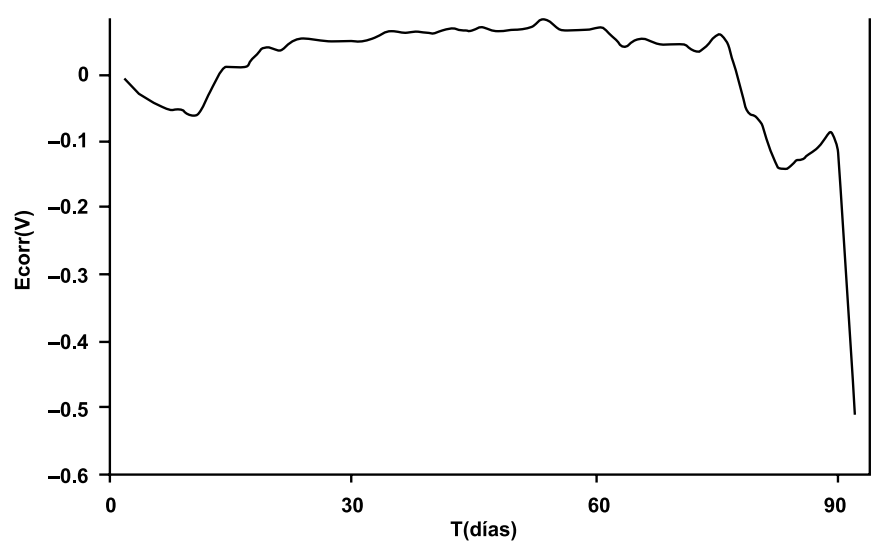

Figura 2. Evolución del potencial de corrosión frente al tiempo de una muestra sumergida en disolución de $\mathrm{NaCl}$ al 3.5\%.

el agua y el oxígeno han alcanzado la interfase metal-pintura y crean unas condiciones alcalinas, debido a la reducción del oxígeno, comenzando el proceso de delaminación [3]. En los últimos días del ensayo, el potencial de corrosión toma valores de oxidación del acero desnudo en $\mathrm{NaCl}$ al 3.5\%, lo que indica que la pintura se degrada casi totalmente $y$ pierde sus propiedades resistivas.

\subsection{Polarización lineal.}

La Figura 3 recoge diversas curvas LP registradas tras distintos tiempos de inmersión en $\mathrm{NaCl}$ de una muestra pintada. Como referencia se han incluido el correspondiente a una muestra sin pintar. Como puede observarse, la intensidad anódica límite para la muestra recién pintada, $i_{\mathrm{m}^{\prime}}$ es varios órdenes de magnitud inferior a la que se dispone para el metal desnudo, lo que da idea de su nivel de protección. Tras 24 horas de ensayo, $\mathrm{i}_{\mathrm{m}}$ aumenta dos órdenes de magnitud, mostrando que el substrato metálico se está oxidando bajo la pintura. Este valor se ve aumentado tras 72 horas de inmersión y de nuevo al final tras 90 días de ensayo hasta valores de intensidad similares a la del metal desnudo. De acuerdo con lo comentado anteriormente, las medidas reflejan que la pintura pierde su propiedad protectora tras un periodo de tiempo prolongado en inmersión.

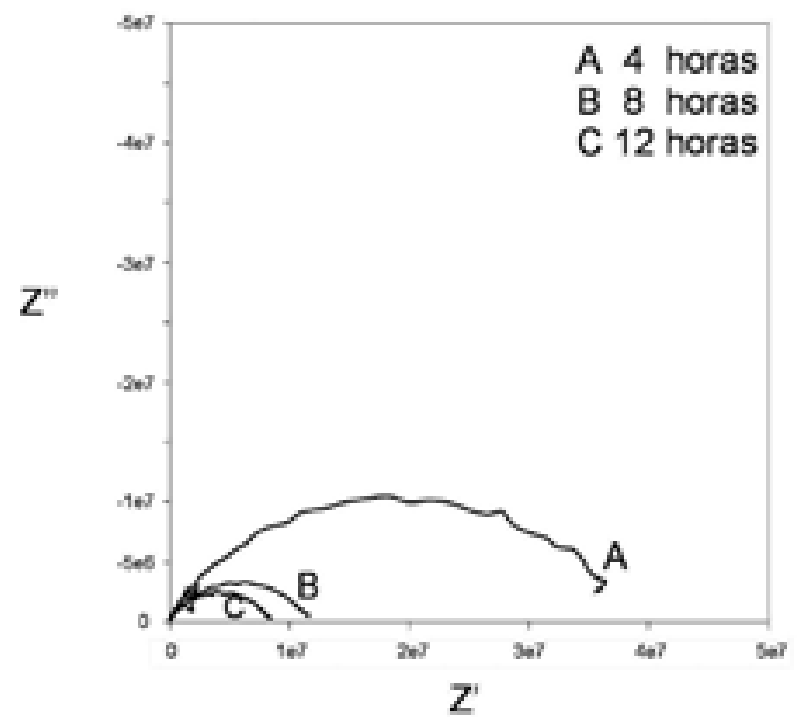

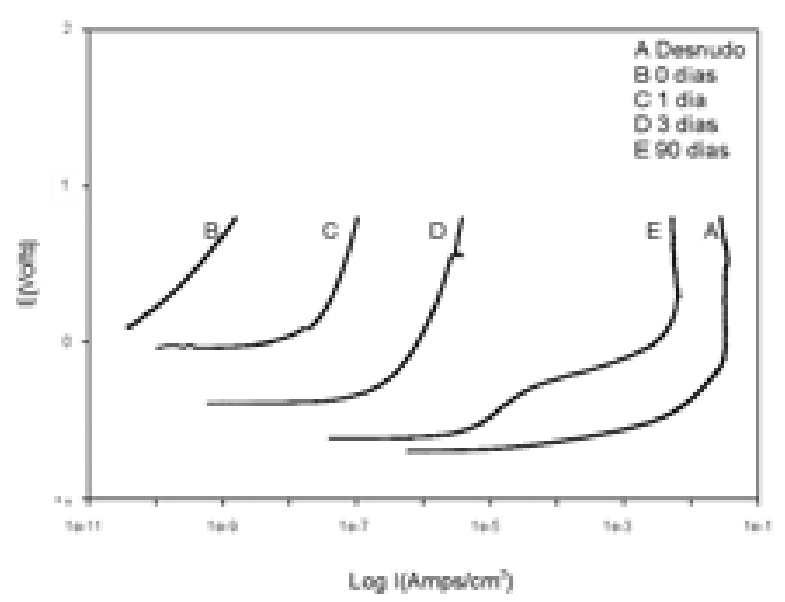

Figura 3. Curvas de polarización lineal registradas a los tiempos de inmersión que se indican en disolución de $\mathrm{NaCl}$ al 3.5\%.

\subsection{Espectroscopía de Impedancia Electroquímica.}

En la Figura 4 se incluyen distintos diagramas EIS correspondientes a ensayos realizados sobre muestras pintadas tras ser sometidas a diferentes tiempos de exposición en la disolución de $\mathrm{NaCl}$. Como puede observarse, en las 12 primeras horas de inmersión se produce una disminución considerable del arco en los diagramas de Nyquist, lo que se atribuye a una disminución de la impedancia, de acuerdo con los valores más activos del potencial de corrosión. Al ajustar los datos al circuito de la Figura 1 se observa cómo se produce un aumento en la capacidad de la pintura y una disminución de la resistencia asociada, Figura 5. De acuerdo con [4], esto sucede cuando la pintura presenta defectos que aceleran la llegada del agua al substrato, aumentando la conductividad de la película polimérica. Esta disminución de $\mathrm{R}_{\mathrm{po}}$ se asocia a que la pintura no posee una buena protección barrera [5]. Así, a partir de las 9 horas, $R_{\mathrm{po}}$ se encuentra por debajo de $10^{7}$, valor por debajo del cual se considera, según criterio general, que la pintura no está protegiendo adecuadamente al substrato metálico [6]. En un principio, la mala protección barrera conferida por parte del recubrimiento se explica por tratarse de una pintura acrílica (polímeros lineales) de base agua con una elevada permeabilidad al electrolito. Sin embargo, los valores de impedancia aumentan tras 4 días de inmersión, Figura 4 ,

Figura 4. Diagramas de Nyquist y Bode registrados a los periodos de inmersión que se indican en disolución de $\mathrm{NaCl}$ al $3.5 \%$. 
y el valor de $\mathrm{R}_{\mathrm{po}}$ lega hasta $10^{7}$ a los 16 días de ensayo. Esta circunstancia se asocia a que, tras elevados tiempos de exposición, los defectos de la película se bloquean con los productos de corrosión formados por la disolución del pigmento anticorrosivo, con lo que aumenta la resistencia del poro [7]. Finalmente, y tras 92 días de ensayo, en la pintura continúan formándose más vías de conducción electrolítica, que no pueden ser tamponadas debido al agotamiento del pigmento. Entonces, $\mathrm{R}_{\mathrm{po}}$ disminuye drásticamente, alcanzando valores menores de $10^{3} \Omega \mathrm{cm}^{2}$, valor por el que se considera que la pintura ya no ejerce efecto protector alguno sobre el metal.

La protección de barrera que presente la pintura dependerá del tiempo de saturación de la entrada de agua. Este tiempo viene dado por la estabilización del valor de la capacidad, C, de la capa. Como se observa en la Figura 5 este tiempo es de aproximadamente 12 horas. Por otro lado, haciendo uso de la relación de Brasher y Kinsbury [2], se calcula el porcentaje de entrada de agua $(\phi)$ hasta el tiempo de saturación, Figura 6. En el sistema estudiado $\phi$ no varía linealmente con $\sqrt{t}$, por lo que se considera que el proceso de difusión no es ideal fickiano [8]. En este caso, se calcula el denominado coeficiente de difusión aparente con el tiempo de saturación [7]. El valor de D obtenido es de $5.21 \times 10^{-9} \mathrm{~cm}^{2} \mathrm{~s}^{-1}$, en buen acuerdo con lo recogido en $[7,9]$. Este valor puede considerarse adecuado al tratarse de una pintura acrílica de base agua que, en principio, es menos resistente a este mismo medio. Por otro lado, debido a que posee pigmentos anticorrosivos que requieren del electrolito para proteger, la entrada de agua en la pintura podría ser en principio favorecida.

\section{CONCLUSIONES}

Se han estudiado las propiedades protectoras de una pintura base agua, de nueva formulación y respetable con el medio ambiente, aplicada sobre un acero de uso naval. De acuerdo con los datos obtenidos, el periodo de saturación de la entrada de agua es de 12 horas, a partir de las cuales, la disolución de los pigmentos mejora las propiedades anticorrosivas de la pintura, mediante el bloqueo de los defectos en la película. Los resultados obtenidos mediante la aplicación de distintas técnicas electroquímicas ponen de manifiesto que el comportamiento del recubrimiento en disoluciones de $\mathrm{NaCl}$ puede considerarse aceptable para un periodo no superior a 90 días. A partir de este tiempo, se degrada completamente y el material queda desprotegido.

\section{AGRADECIMIENTOS}

Este trabajo ha sido financiado por la Comisión Interministerial de Ciencia y Tecnología, el proyecto MAT2001-3477 y por la Junta de Andalucía.

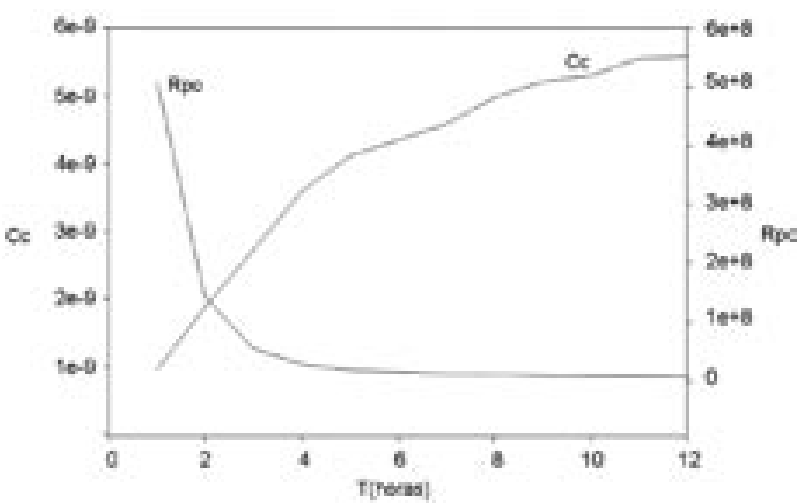

Figura 5. Evolución de la capacidad y de la resistencia de la pintura en función del tiempo inicial de exposición.

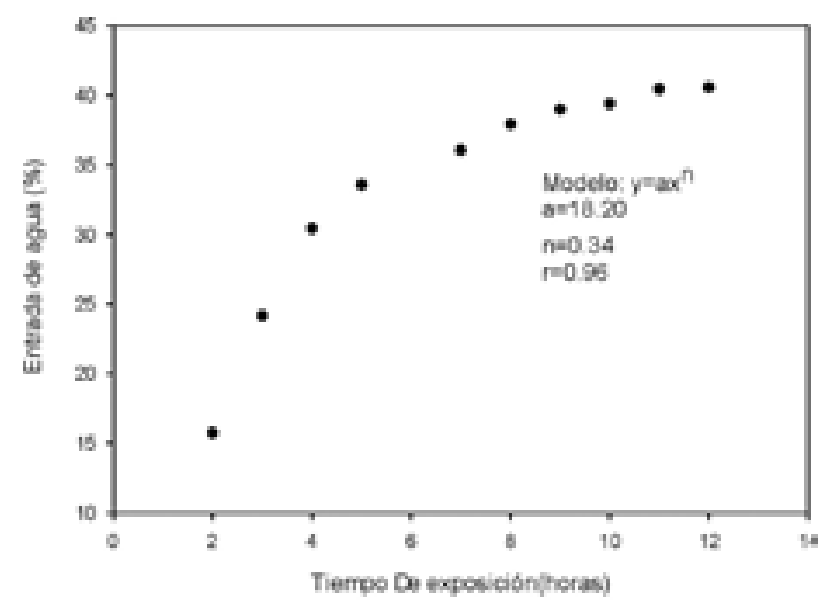

Figura 6. Evolución de la entrada de agua en la pintura estimada a partir de las medidas de EIS.

\section{BIBLIOGRAFÍA}

1. K. Barton, Protection Against Atmospheric Corrosion, Wiley, New York (USA), 1976

2. D.M. Brasher y A.H. Kinsbury, J. Appl. Chem., 4 (1954)62.

3. O. Ferraz, E. Cavalcanti, A.R. di Sarli. Corrosion Science, 37 (1995)1267-1280.

4. M. Marrero, G. Di Filippo, J. Vera. Proc. II Congreso Iberoamericano de Corrosión y Protección (1988) 153-162.

5. A. Di Sarli, CIDEPINT, Anales (1995) 181-253.

6. D.J. Mills. Corrosion Management, July/August (2000) 10-15.

7. M.C. Perez Perez, Tesis doctoral, Universidad de Vigo, 1998.

9. M.M. Wind, H.J.W. Lenderink, Progress in Organic Coatings, 28 (1996)239250

10. A. Amiridium, D. Thierry. British Corrosion J., 30(2) (1995) 128.

Recibido: 1.2 .03

Aceptado: 30.11 .03 